\title{
Inflammation and pro-resolution inflammation after hepatobiliary surgery
}

Juan P. Cata ${ }^{1 *}$, Jose F. Velasquez², Maria F. Ramirez³ , Jean-Nicolas Vauthey ${ }^{4}$, Vijaya Gottumukkala', Claudius Conrad ${ }^{4}$ Bradford J. Kim ${ }^{4}$ and Thomas Aloia ${ }^{4}$

\begin{abstract}
Background: The magnitude of the perioperative inflammatory response plays a role in surgical outcomes. However, few studies have explored the mechanisms of the resolution of inflammation in the context of surgery. Here, we described the temporal kinetics of interleukin-6, cortisol, lipoxin A4, and resolvin D in patients who underwent oncologic liver resections.
\end{abstract}

Methods: All patients gave written informed consent. Demographic and perioperative surgical data were collected, along with blood samples, before surgery and on the mornings of postoperative days 1,3 , and 5. Interleukin-6, cortisol, lipoxin-A4, and resolvin D were measured in plasma. A $P$ value $<0.05$ was considered statistically significant.

Results: Forty-one patients were included in the study. Liver resection for colorectal metastatic disease was the most commonly performed surgery. The plasma concentrations of interleukin- 6 were highest on day 1 after surgery and remained higher than the baseline up to postoperative day 1. Postoperative complications occurred in 14 (24\%) patients. Cortisol concentrations spiked on postoperative day 1. The concentrations of lipoxin A4 and resolvin D were lowest on day 1 after surgery.

Conclusions: The inflammatory response associated with hepatobiliary surgery is associated with low circulating concentrations of lipoxin A4 and resolvin D that mirror, in an opposite manner, the kinetics of interleukin 6 and cortisol.

Trial registration: NCT01438476

Keywords: Inflammation, Resolution, Hepatobiliary surgery, Complications

\section{Background}

Increasing evidence indicates that inflammation has a significant impact on short- and long-term postoperative clinical outcomes. Several studies have found high circulating concentrations of interleukin-6 (IL-6) or Creactive protein in patients who develop postoperative complications or die within 30 days of surgery [1, 2]. Kimura et al. demonstrated that patients who developed postoperative infectious complications after liver resection had significantly higher concentrations of IL- 6 on days 0 and 1 after surgery than did those without infections [1].

\footnotetext{
* Correspondence: jcata@mdanderson.org

${ }^{1}$ Department of Anesthesiology and Perioperative Medicine, The University

of Texas MD Anderson Cancer Center, Houston, TX 77030, USA

Full list of author information is available at the end of the article
}

Specialized pro-resolvin (Rv) mediators (SPMs) such as lipoxin A4 (LXA4), E-D series Rvs, protectins, and maresins are released in response to tissue injury and actively promote the resolution of inflammation [3]. Lipoxins and Rvs are formed in a process known as transcellular biosynthesis, which uses arachidonic acid and $\alpha-3$ fatty acids as substrates [3]. Lipoxins and Rvs appear to be important mediators of the surgical stress response. In rodents, surgery-induced release of IL-6 was inhibited by aspirin-triggered RvD1 [4]. In an experimental model of peritonitis, the administration of fish oil (a natural source of eicosapentaenoic and docosahexaenoic acids) decreased the levels of IL-6 [5]. In patients who were undergoing major vascular surgery, two different patterns of inflammation and resolution were described on the basis of the temporal kinetics of 
SPMs: a pro-inflammatory pattern and a pro- $\mathrm{Rv}$ mediator profile [6].

To date, the temporal kinetics of SPMs in cancer patients who undergo major surgery remains unknown. Thus, in this study, we measured the plasma concentrations of IL-6, cortisol, LXA4, and RvD in patients who underwent oncologic hepatobiliary surgery. We hypothesized that low plasma concentrations of SPMs would be observed after surgery, particularly in patients who developed postoperative complications.

\section{Methods}

\section{Ethics, consent, and permissions}

After receiving institutional review board (IRB \#20111046) approval and obtaining written informed consent from all patients, we collected demographic and perioperative data from an ongoing randomized controlled study of hepatobiliary surgery for primary biliary cancer or metastatic liver cancer (NCT01438476) at the University of Texas MD Anderson Cancer Center (Houston, Texas). We included all patients who underwent surgery between January 2013 and March 2015and were 18 years of age or older. We excluded patients who underwent emergency surgeries, those who were taking opioids chronically, pregnant women, and patients with major psychiatric conditions or immunologic disorders.

The anesthesia technique and postoperative pain management used in these patients was described elsewhere [7]. In brief, all patients underwent general balanced anesthesia, with or without postoperative epidural analgesia or intravenous patient-controlled analgesia. Surgical complication data were collected in real time and scored using the Accordion Severity Grading System [8]. Each complication was categorized into its appropriate system: neurology, cardiovascular, pulmonary, endocrine, metabolic, gastrointestinal, renal, hematology, wound, sepsis, and others. In addition, post-operative bile leak and liver failure were included for analysis using standard definitions $[9,10]$. All complications and grades were verified by two staff members of the surgical oncology department at our institution.

Blood specimens were collected before surgery and on the mornings of postoperative days 1, 3, and 5. IL-6, cortisol, LXA4, and RvD concentrations in plasma were measured using an enzyme-linked immunosorbent assay and commercially available kits (R\&D Systems, Inc., Minneapolis, CA, and MyBioSource, Inc., San Diego, CA). The assays were read in triplicate using an automated microplate reader (Molecular Devices, Inc., Sunnyvale, CA).

\section{Sample size calculation and statistical analysis}

On the basis of previously published data [11] and using a type I error rate $(\alpha)$ of $5 \%$ and a power $(1-\beta)$ of $80 \%$, we estimated that 41 patients would be needed to demonstrate an increase of at least $50 \%$ in the plasma concentrations of IL- 6 on day 1 after surgery.

Demographic, intraoperative, and postoperative data were analyzed and summarized using medians (interquartile ranges [IQRs]) or means (standard deviations or 95\% confidence intervals) to account for outliers and non-normality in our description of study variables. The chi-square or Fisher's exact test was used to compare categorical variables. The Friedman test, followed by Dunn's multiple comparisons test, was used to compare preoperative and postoperative concentrations of IL-6, cortisol, LXA4, and RvD in the overall group of patients, and the Kruskal-Wallis test, followed by Dunn's multiple comparisons test, was used to assess perioperative differences between patients with and without postoperative complications. A $P$ value $<0.05$ was considered statistically significant. The Spearman correlation test was used to assess the correlation between the concentrations of SMPs and IL-6. Prism 5 software (GraphPad Software, Inc., San Diego, CA) was used for all statistical analyses.

\section{Results}

\section{Patients}

Forty-one patients (22 male [54\%]) with a median age of 57 years were included in the study (Table 1). Forty (98\%) had an ASA physical status of 3. The median [IQR] heights and weights were 170 [163-176.5] $\mathrm{cm}$ and 78 [64.75-90.4] kg. Liver resection $(n=40$ [98\%]) for colorectal metastatic disease $(n=25[61 \%])$ was the most common surgical procedure. Fourteen patients (44\%) developed postoperative complications; however, in 9, they were classified as moderate or severe (Accordion $\geq 2$ ) (Table 2).

\section{Laboratory results}

As shown in Fig. 1a, the median (IQR) plasma concentrations of IL-6 were significantly higher on days 1 (216 [55.5-421] ng/mL, $p<0.001), 3$ (128 [48.5-279] ng/mL), and 5 (70 [14-204] ng/mL, $p<0.001)$ after surgery than preoperatively $(28[2.5-109] \mathrm{ng} / \mathrm{mL}, p=0.001)$. A Kruskal-Wallis test showed that the perioperative concentrations of IL-6 were slightly higher in patients with postoperative complications than in those without. However, no statistically significant differences were found for individual days (Table 3).

The concentrations of cortisol also increased after surgery (Fig. 1b), but only those measured on day 1 (39.32 [29.74-78.3] $\mathrm{pg} / \mathrm{mL}, p=0.04$ ) were statistically significantly higher than those measured before surgery (preoperative: 33.17 [15.81-46.42] $\mathrm{pg} / \mathrm{mL}$ vs. postoperative day 3: $34.74[17.45-51.19] \mathrm{pg} / \mathrm{dL}, p=0.999$ and vs. postoperative day 5: 36.2 [14.78-48.83] pg/dL, $p=0.837)$. Patients with and without moderate or severe postoperative 
Table 1 Demographic and perioperative variables among 41 patients who underwent hepatobiliary surgery

\begin{tabular}{|c|c|}
\hline Variable & Result \\
\hline Age, years [IQR] & $57[48.5-67.5]$ \\
\hline Sex, female/male, $n(\%)$ & $22(54) / 19(46)$ \\
\hline Height, cm [IQR] & $170[163-176.5]$ \\
\hline Weight, kg [IQR] & $78[64.75-90.4]$ \\
\hline \multicolumn{2}{|l|}{ ASA physical status, $n(\%)$} \\
\hline 1 or 2 & $1(2)$ \\
\hline 3 or 4 & $40(98)$ \\
\hline \multicolumn{2}{|l|}{ Comorbidities, yes/no, n (\%) } \\
\hline Coronary artery disease & $1(2) / 40(98)$ \\
\hline Hypertension & $11(27) / 30(73)$ \\
\hline Hyperlipemia & $4(10) / 37(90)$ \\
\hline Diabetes mellitus & $3(7) / 38(93)$ \\
\hline Hypothyroidism & $4(10) / 37(90)$ \\
\hline \multicolumn{2}{|l|}{ Medications, n (\%) } \\
\hline Statins & $5(12) / 36(88)$ \\
\hline Antihypertensives & $13(31) / 28(69)$ \\
\hline Antidiabetic drugs & $3(7) / 38(93)$ \\
\hline Non-steroidal anti-inflammatory drugs & $2(5) / 39(95)$ \\
\hline \multicolumn{2}{|l|}{ Histology, n (\%) } \\
\hline Cholangiocarcinoma & $25(61)$ \\
\hline Gallbladder carcinoma & $4(10)$ \\
\hline Colorectal carcinoma & $2(5)$ \\
\hline Other & $10(24)$ \\
\hline Preoperative chemotherapy, yes/no, $n$ (\%) & $27(66) / 19(34)$ \\
\hline \multicolumn{2}{|l|}{ Type of surgery, $n$ (\%) } \\
\hline Liver resection & $40(98)$ \\
\hline Pancreatectomy & $1(2)$ \\
\hline Duration of surgery (min), median [range] & $293[219.5-340.5]$ \\
\hline
\end{tabular}

Continuous variables are shown as medians [IQR, interquartiles]

$\mathrm{Cm}$ centimeters, $\mathrm{kg}$ kilograms, ASA American Society of Anesthesiologists

complications had similar perioperative plasma concentrations of cortisol (Table 3).

The circulating levels of LXA4 appear to mirror, in an opposite manner, those of IL-6. In brief, the median (IQR) plasma concentrations of LXA4 were significantly lower on postoperative days $1(3.94[1.36-7.45] \mathrm{ng} / \mathrm{mL}$, $p<0.001), 3(4.71[1.61-9.39] \mathrm{ng} / \mathrm{mL}, p<0.001)$, and 5 $(5.02[1.83-8.68] \mathrm{ng} / \mathrm{mL}, p=0.006)$ than before surgery (7 [1.94-15.98] ng/mL). The Spearman correlation test demonstrated a significantly negative correlation $(r=-$ 0.5 [95\% confidence interval -0.63 to 0.35 ],$p<0.0001$ ) between the serum concentrations of IL- 6 and LXA4. The postoperative concentrations of LXA4 were slightly but not statistically significantly higher in patients without complications than in those with moderate or severe (Accordion $\geq 2$ ) complications (Table 3).
Table 2 Postoperative complications among 14 patients who underwent hepatobiliary surgery

\begin{tabular}{ll}
\hline Variable & Result \\
\hline Estimated blood loss, mL [IQR] & $250[150-450]$ \\
Red blood cell transfusion, yes/no, $n$ (\%) & \\
Intraoperative & $1(2) / 40(98)$ \\
Postoperative & $5(12) / 36(88)$ \\
Complication type, yes/no, $n$ (\%) & \\
Neurologic & $1(2) / 40(98)$ \\
Cardiac & $1(2) / 40(98)$ \\
Respiratory & $1(2) / 40(98)$ \\
Renal & $0(0) / 41(100)$ \\
Gastrointestinal & $4(10) / 37(90)$ \\
Wound & $2(5) / 39(95)$ \\
Deep venous thrombosis/pulmonary embolism & $2(5) / 39(95)$ \\
Other & $3(7) / 38(93)$ \\
Highest Accordian grade, $n$ (\%) & \\
1 & $5(36 \%)$ \\
2 & $5(36 \%)$ \\
3 & $4(28 \%)$ \\
\hline IQR interquartiles &
\end{tabular}

Similar to the kinetics followed by LXA4, we found that in comparison to preoperative values $(0.88$ [0.66$1.44 \mathrm{ng} / \mathrm{mL}$ ), there was a significant decrease in the concentrations of $\mathrm{RvD}$ on day 1 (0.63 [0.39-0.81] ng/ $\mathrm{mL}, p<0.001)$ after surgery, but not on days $3(0.79$ [0.63-1.01] ng/mL, $p=0.298)$ and 5 (0.84 [0.66$1.29 \mathrm{ng} / \mathrm{mL}, p=0.999$ ) (Fig. $2 \mathrm{~b}$ ). In addition, a significant negative correlation $(r=-0.39$ [95\% confidence interval -0.63 to 0.08 ] $p=0.11$ ) was found between the serum concentrations of IL- 6 and RvD. Although patients with moderate or severe (Accordion $\geq 2$ ) complications had slightly lower $\mathrm{RvD}$ concentrations on postoperative days 1,3 , and 5 (Table 3), these differences were not statistically significant.

\section{Discussion}

In our study, we found markers of inflammation and neuroendocrine stress response after surgery for primary biliary cancer or metastatic liver cancer, as evidenced by an increase in the plasma concentrations of IL- 6 and cortisol.

Other investigators have demonstrated that IL-6 peaks after hepatobiliary surgery [12]. We found that IL-6 peaked on day 1 after surgery; however, there were no significant differences in the concentrations of cytokines between patients with and without complications. Conversely, Lahiri et al. demonstrated that the serum concentrations of IL-6 were statistically significantly different in patients with and without systemic inflammatory response 


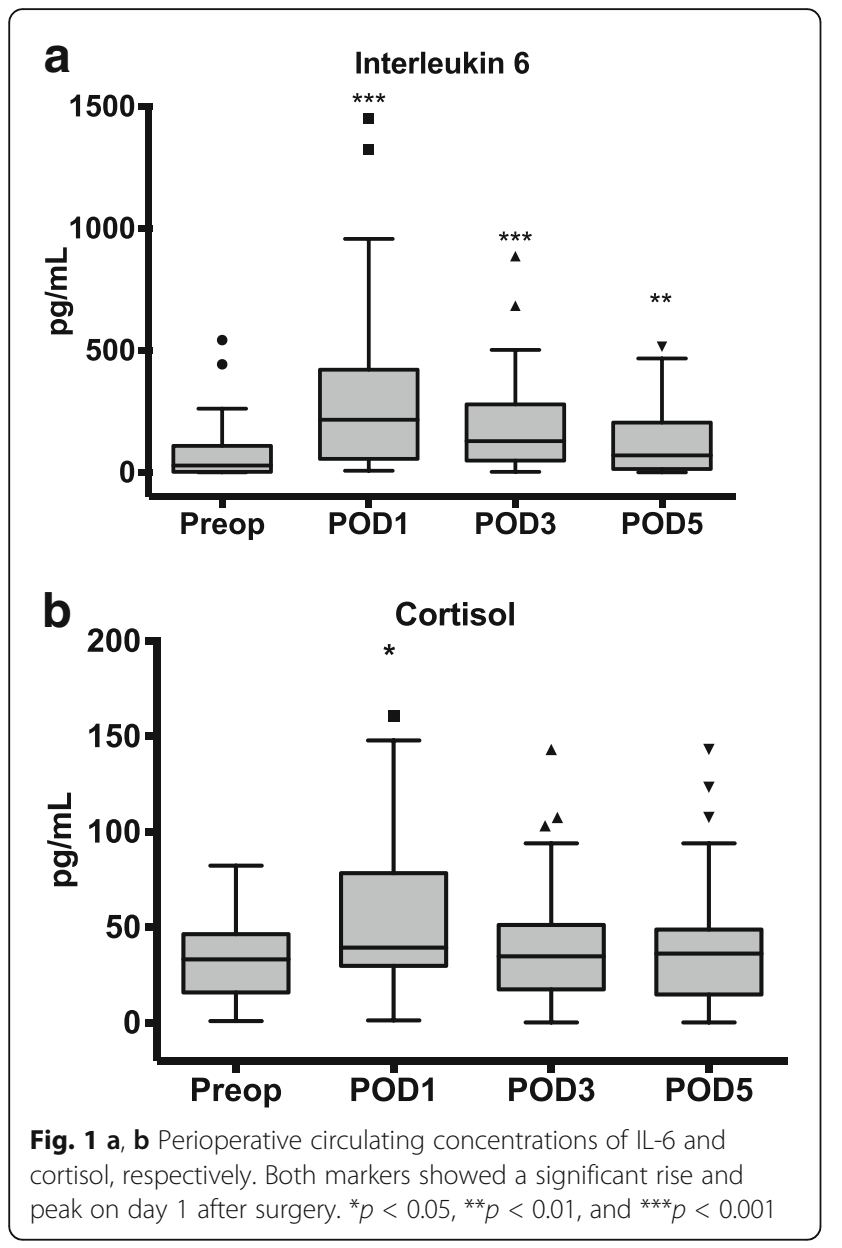

syndrome after hepatobiliary surgery [12]. The discrepancies in the findings between Lahiri et al.'s work and ours are presumably due to differences in blood specimen collection days, outcome definitions, and unknown variables such as the anesthesia and analgesia technique used during and after surgery.

To the best of our best knowledge, this is the first study to investigate the temporal kinetics of LXA4 and $\mathrm{RvD}$ in oncologic patients undergoing surgery. In brief, we found that the plasma concentrations of LXA4 and RvD mirrored the perioperative kinetics of IL- 6 and cortisol in a reverse direction. Specifically, the concentrations of LXA4 and RvD had reached their nadir on postoperative day 1 , which coincided with the peaks in IL- 6 and cortisol. The relevance of these findings remains unclear. It is possible that the initial postoperative decrease in plasma LXA4 and RvD, occurring at the same time as the peak in IL-6, is part of the stress response to surgical trauma (which is more intense than the lipopolysaccharide challenge), while the return to preoperative values represents the resolutive phase of surgery-induced trauma.

LXA4 and RvD are newly discovered molecules that function in the resolution of acute inflammation, pain, and sepsis. Both SPMs have important roles in a cascade of events that occur as part of the inflammatory and immune-suppressive response during and after surgery. For instance, LXA4 inhibits chemotaxis, neutrophil-epithelial cell interaction, natural killer cell function, and the radical of oxygen formation, while

Table 3 Plasma concentrations of IL-6, cortisol, LXA4, and RvD in patients with and without postoperative complications

\begin{tabular}{|c|c|c|c|c|}
\hline Cytokine & Median preoperative [IQR] & Median POD-1 [IQR] & Median POD-3 [IQR] & Median POD-5 [IQR] \\
\hline \multicolumn{5}{|l|}{$\mathrm{IL}-6, \mathrm{pg} / \mathrm{mL}$} \\
\hline Complications, $n=9$ & 34 [19.33-46.25] & 254 [82.25-593.3] & $98[54.75-268.3]$ & $76[50-284.8]$ \\
\hline No complications, $n=31$ & 27 [0-115] & 206 [38-359] & 128 [44-286] & 59 [3-163] \\
\hline$P$ value & 0.999 & 0.999 & 0.999 & 0.991 \\
\hline \multicolumn{5}{|l|}{ Cortisol, pg/mL } \\
\hline Complications, $n=9$ & $28.1[13.60-40.66]$ & $34.75[31.19-87.6]$ & $27.72[8.46-46.2]$ & $30.88[16.4-48.3]$ \\
\hline No complications, $n=29$ & $33.34[16.8-54.37]$ & 40 [25.69-81.35] & 39.12 [17.96-51.78] & $40.36[13.52-56.21]$ \\
\hline$p$ value & 0.999 & 0.999 & 0.999 & 0.999 \\
\hline \multicolumn{5}{|l|}{ LXA4, pg/mL } \\
\hline Complications, $n=9$ & $3.95[1.57-19.52]$ & $3.2[1.05-6.25]$ & $3.15[1.12-8.63]$ & $2.87[1.23-6.01]$ \\
\hline No complications, $n=31$ & $7[2.01-17.24]$ & $4.21[1.48-8.99]$ & $4.93[1.68-10.64]$ & $5.96[2.27-13.63]$ \\
\hline$p$ value & 0.999 & 0.968 & 0.999 & 0.534 \\
\hline \multicolumn{5}{|l|}{$\mathrm{RvD}, \mathrm{pg} / \mathrm{mL}$} \\
\hline Complications, $n=9$ & $1.13[0.56-1.85]$ & $0.43[0.28-1.5]$ & $0.76[0.28-1.03]$ & $1.02[0.48-1.39]$ \\
\hline No complications, $n=27$ & $0.89[0.6-1.5]$ & $0.68[0.47-1.03]$ & $0.98[0.67-1.93]$ & $1.01[0.69-2]$ \\
\hline$p$ value & 0.999 & 0.435 & 0.501 & 0.999 \\
\hline
\end{tabular}

IL-6 interleukin 6, LXA4 lipoxin A4, RvD resolvin D, IQR interquartile range, $P O D$ postoperative day 


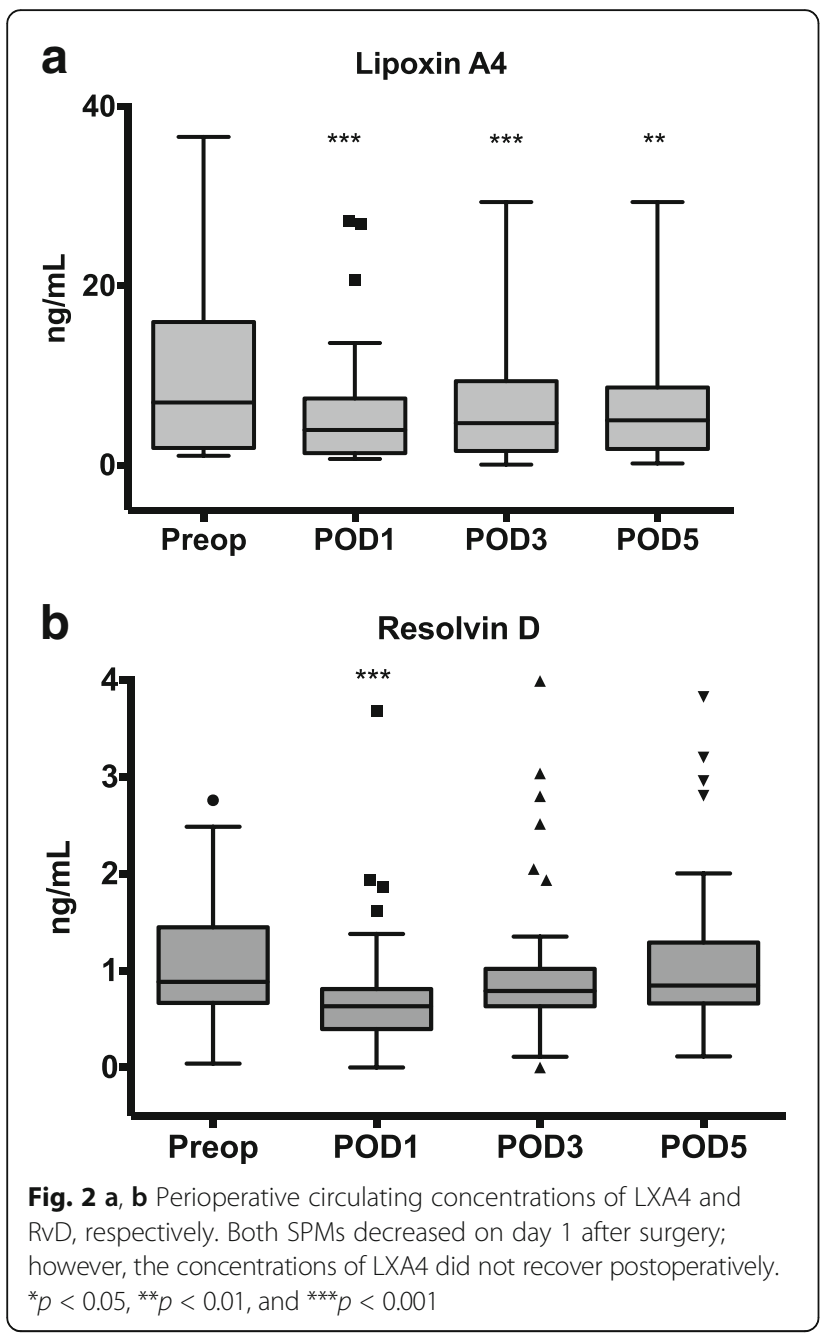

RvD is able to reduce the migration of neutrophils and the production of tumor necrosis factor [3]. Furthermore, there is increasing evidence that LXA4 has protective effects in animal models of systemic inflammation [13]. As an example, the administration of LXA4 in rodents with lipopolysaccharide-induced systemic inflammation significantly decreased the plasma concentration of IL-6 [13].

The roles that LXA4 and RvD play in recovery after surgery are largely unknown. Persistent low concentrations of SPMs after vascular surgery suggest a failure to resolve the inflammatory response and the presence of postoperative complications [6]. We were not able to show that patients who develop postoperative complications have higher plasma of levels of IL- 6 and cortisol or lower circulating concentrations of SPMs than do those with an uneventful recovery. However, it is worth mentioning that our study was underpowered to show statistically significant differences in patients with and without complications. Also, we might have missed the nadirs or peaks of SPMs in our patients, with and without complications, because their timing depends on individual time courses of inflammation [14].

This study has several other limitations. First, we measured the levels of LXA4 and RvD using ELISA. It is well accepted that this is not the gold standard assay for determination of these molecules; however, a recent study by Fedirko et al. demonstrated a good correlation between the concentrations of LXA4 and RvD measured by spectrometry and ELISA (correlations of 0.88 and 0.83 , respectively). Second, we only investigated the temporal kinetics of LXA4 and RvD. Therefore, the perioperative kinetics of other SPMs, such as RvE, protectins, and maresins, and their significance in postoperative recovery and relationship with morbidity, remain to be studied. Third, we included patients with a variety of cancers, comorbidities, and surgeries, which can influence the plasma concentrations of both proinflammatory cytokines and SPMs. We did not include a control group of subjects to compare their concentrations of LXA4 and RvD with those measured in our population of patients. Lastly, we did not consider the effects of drugs that can modulate the kinetics of SPMs, such as thiazolidinedione, aspirin, and fish oil, or the individual effects of different anesthetic techniques [15-17].

\section{Conclusions}

The results of the present study demonstrate that the inflammatory response associated with hepatobiliary surgery is associated with low circulating concentrations of LXA4 and RvD. Understanding the profiles of the molecules that function in the resolution of inflammation can help identify patients who are likely to experience poor postoperative recovery. More studies are needed to elucidate the clinical relevance of these findings, particularly in the context of recovery after surgery.

\section{Acknowledgements \\ Not applicable}

\section{Funding}

No funding body provided support for the design of the study; the collection, analysis, and interpretation of data; or the writing of the manuscript.

\section{Availability of data and materials}

Please contact the corresponding author for data requests.

\section{Authors' contributions}

JPC made the study design, data collection and interpretation, and manuscript writing. JFV, MFR, and BJK participated in the data collection. TA contributed in the study design. JNV, VG, CC, and TA, did the final approval of the manuscript. All authors read and approved the final manuscript.

Ethics approval and consent to participate

This study was approved by the MD Anderson Institutional Review Board (IRB \#2011-1046). All patients gave written informed consent prior to enrolling in the study. 


\section{Consent for publication}

Not applicable

\section{Competing interests}

The authors declare that they have no competing interests.

\section{Publisher's Note}

Springer Nature remains neutral with regard to jurisdictional claims in published maps and institutional affiliations.

\section{Author details}

'Department of Anesthesiology and Perioperative Medicine, The University of Texas MD Anderson Cancer Center, Houston, TX 77030, USA. ${ }^{2}$ CES University, Antioquia, Colombia. ${ }^{3}$ Department of Anesthesiology, Massachusetts General Hospital, Harvard University, Boston, MA, USA. ${ }^{4}$ Department of Surgical Oncology, The University of Texas MD Anderson Cancer Center, Houston, TX, USA.

Received: 9 March 2017 Accepted: 5 August 2017

Published online: 10 August 2017

\section{References}

1. Kimura F, Shimizu H, Yoshidome H, Ohtsuka M, Kato A, Yoshitomi H, Nozawa S, Furukawa K, Mitsuhashi N, Sawada S, et al. Circulating cytokines, chemokines, and stress hormones are increased in patients with organ dysfunction following liver resection. J Surg Res. 2006;133:102-12.

2. Blakely AM, Heffernan DS, McPhillips J, Cioffi WG, Miner TJ. Elevated Creactive protein as a predictor of patient outcomes following palliative surgery. J Surg Oncol. 2014;110:651-5.

3. Basil MC, Levy BD. Specialized pro-resolving mediators: endogenous regulators of infection and inflammation. Nat Rev Immunol. 2016;16:51-67.

4. Terrando N, Gomez-Galan M, Yang T, Carlstrom M, Gustavsson D, Harding RE, Lindskog M, Eriksson LI. Aspirin-triggered resolvin D1 prevents surgeryinduced cognitive decline. FASEB J. 2013;27:3564-71.

5. Tomasdottir V, Vikingsson A, Freysdottir J, Hardardottir I. Dietary fish oil reduces the acute inflammatory response and enhances resolution of antigen-induced peritonitis. J Nutr Biochem. 2013:24:1758-65.

6. Pillai PS, Leeson S, Porter TF, Owens CD, Kim JM, Conte MS, Serhan CN, Gelman S. Chemical mediators of inflammation and resolution in postoperative abdominal aortic aneurysm patients. Inflammation. 2012;35:98-113.

7. Ramirez MF, Ai D, Bauer M, Vauthey JN, Gottumukkala V, Kee S, Shon D, Truty M, Kuerer HM, Kurz A, et al. Innate immune function after breast, lung, and colorectal cancer surgery. J Surg Res. 2015;194:185-93.

8. Porembka MR, Hall BL, Hirbe M, Strasberg SM. Quantitative weighting of postoperative complications based on the accordion severity grading system: demonstration of potential impact using the american college of surgeons national surgical quality improvement program. J Am Coll Surg. 2010:210:286-98.

9. Brooke-Smith M, Figueras J, Ullah S, Rees M, Vauthey JN, Hugh TJ, Garden OJ, Fan ST, Crawford M, Makuuchi M, et al. Prospective evaluation of the International Study Group for Liver Surgery definition of bile leak after a liver resection and the role of routine operative drainage: an international multicentre study. HPB (Oxford). 2015;17:46-51.

10. Rahbari NN, Garden OJ, Padbury R, Brooke-Smith M, Crawford M, Adam R, Koch M, Makuuchi M, Dematteo RP, Christophi C, et al. Posthepatectomy liver failure: a definition and grading by the International Study Group of Liver Surgery (ISGLS). Surgery. 2011;149:713-24.

11. Erinjeri JP, Thomas CT, Samoilia A, Fleisher M, Gonen M, Sofocleous CT, Thornton RH, Siegelbaum RH, Covey AM, Brody LA, et al. Image-guided thermal ablation of tumors increases the plasma level of interleukin- 6 and interleukin-10. J Vasc Interv Radiol. 2013;24:1105-12.

12. Lahiri R, Derwa Y, Bashir Z, Giles E, Torrance HD, Owen HC, O'Dwyer MJ, O'Brien A, Stagg AJ, Bhattacharya S, et al. Systemic inflammatory response syndrome after major abdominal surgery predicted by early upregulation of TLR4 and TLR5. Ann Surg. 2016;263:1028-37.

13. Cheng Q, Wang Z, Ma R, Chen Y, Yan Y, Miao S, Jiao J, Cheng X, Kong L, Ye D. Lipoxin A4 protects against lipopolysaccharide-induced sepsis by promoting innate response activator B cells generation. Int Immunopharmacol. 2016;39: 229-35.
14. Martindale RG, Warren MM, McClave SA. Does the use of specialized proresolving molecules in critical care offer a more focused approach to controlling inflammation than that of fish oils? Curr Opin Clin Nutr Metab Care. 2016:19:151-4.

15. Gutierrez AD, Sathyanarayana $P$, Konduru S, Ye Y, Birnbaum Y, Bajaj M. The effect of pioglitazone treatment on 15-epi-lipoxin A4 levels in patients with type 2 diabetes. Atherosclerosis. 2012;223:204-8.

16. Brezinski DA, Nesto RW, Serhan CN. Angioplasty triggers intracoronary leukotrienes and lipoxin A4. Impact of aspirin therapy. Circulation. 1992;86:56-63.

17. Grenon SM, Owens CD, Nosova EV, Hughes-Fulford M, Alley HF, Chong K, Perez S, Yen PK, Boscardin J, Hellmann J, et al. Short-term, high-dose fish oil supplementation increases the production of omega-3 fatty acid-derived mediators in patients with peripheral artery disease (the OMEGA-PAD I Trial). J Am Heart Assoc. 2015:4:e02034.

\section{Submit your next manuscript to BioMed Central and we will help you at every step:}

- We accept pre-submission inquiries

- Our selector tool helps you to find the most relevant journal

- We provide round the clock customer support

- Convenient online submission

- Thorough peer review

- Inclusion in PubMed and all major indexing services

- Maximum visibility for your research

Submit your manuscript at www.biomedcentral.com/submit
C Biomed Central 